\title{
An intrinsic characterization of spherically symmetric spacetimes
}

\author{
Joan Josep Ferrando ${ }^{1}$ and Juan Antonio Sáez ${ }^{2}$ \\ ${ }^{1}$ Departament d'Astronomia i Astrofísica, Universitat de València, E-46100 \\ Burjassot, València, Spain. \\ 2 Departament de Matemàtiques per a l'Economia i l'Empresa, Universitat de \\ València, E-46071 València, Spain \\ E-mail: joan.ferrando@uv.es; juan.a.saez@uv.es
}

\begin{abstract}
We give the necessary and sufficient (local) conditions for a metric tensor to be a non conformally flat spherically symmetric solution. These conditions exclusively involve explicit concomitants of the Riemann tensor. As a direct application we obtain the ideal labeling of the Schwarzschild, Reissner-Nordström and LemaitreTolman-Bondi solutions.

PACS numbers: 04.20.C, 04.20.-q
\end{abstract}

\section{Introduction}

The spherically symmetric solutions of the Einstein equation have played an essential role in further development of the General Relativity theory. The simplicity of their geometry has allowed analytic developments in modeling several astrophysical scenarios: the Schwarzschild and Reissner-Nordström solutions as the gravitational field exterior to a compact object, the geodesic motion in the Schwarzschild geometry explaining the perihelion advance and the bending of light, the relativistic equations of stellar structure in spherically symmetric perfect fluid solutions, the Friedmann-Lemaitre-RobertsonWalker cosmological models, among others (see, e.g., [1]).

The spherically symmetric solutions continue being a subject of study and, moreover, interest in them is increasing nowadays. For instance, in numerical relativity they are an important tool in testing fully general-relativistic time-dependent numerical codes which evolve matter in strong gravitational fields (see, e.g., [2] an references therein). Nevertheless, despite the extensive literature on spherically symmetric solutions, a fully algorithmic characterization of these spacetimes has not been accomplished. The goal of this paper is to attain such a characterization.

The intrinsic characterization of spherically symmetric spacetimes is an old problem established and partially solved by Takeno in 1952 [3] (see also [4]). In the abstract he 
advanced: "In this note a theory is developed concerning the problem of determining whether or not a space-time defined by a line element arbitrarily given in any coordinate system is spherically symmetric". And in the introduction Takeno adds: "If the answer of this problem is given by the tensor equations to be satisfied by the curvature tensor specially when the equations contain no tensor other than the metric tensor, the metric volume element and the curvature tensor, we may say that the problem is solved in the most desirable form". Finally, in his conclusions he says:" ...a theory concerning the discrimination of the spherically symmetric spacetimes has been constructed. Although it is not of the ideal form,...".

Given Takeno's work and his clear ideas two questions naturally arise: Is it possible to solve the problem of characterizing the spherically symmetric spacetimes in "the ideal form"? Why doesn't Takeno solve the problem in this "most desirable form"?

The answer to the first question is affirmative as a consequence of a result by Cartan [5] who showed that each Riemannian geometry may be characterized in terms of the Riemann tensor and its covariant derivatives. Nevertheless, the labeling of metrics in the "ideal form" starts with the beginning of the Riemannian geometry. The characterization of locally flat Riemann spaces [6], Riemann spaces with a maximal group of isometries [7], and locally conformally flat Riemann spaces [8] [9] [10] was achieved before 1921. In all these historic results, the conditions involve explicit concomitants of the curvature tensor (Riemann, Weyl and Cotton tensors). Then, they are intrinsic (depend solely on the metric tensor $g$ ) and, besides, the explicit expression of these concomitants in terms of $g$ is known. We say that a characterization with these two properties, intrinsic and explicit, is an ideal characterization

It is worth pointing out that an understanding of the algebraic structure of the Weyl and Ricci tensors and the covariant determination of their underlying geometry are necessary tools to characterize spacetimes in an ideal form. But this comprehension of the Riemann tensor had not been attained in the epoch of the Takeno's aforementioned papers, which explains why he could not achieve the characterization of the spherically symmetric spacetimes in an ideal form.

The algebraic classification of the space-time symmetric tensors was given by Churchill [11], and since the sixties a lot of work has been devoted to studying the Ricci tensor from an algebraic point of view (see, e.g., the paper by Plebański [12]). However, the general covariant method to determine the Ricci eigenvectors and their causal character was not published until 1992 [13].

The pioneering papers by Petrov [14] and Bel [15] studied the algebraic classification of the Weyl tensor. Since then several algorithms have been proposed to determine the Petrov-Bel type. However, the general covariant expressions that give the underlying geometry of the Weyl tensor for every Petrov-Bel type were not published until 2001

$\ddagger$ The use of the appellation IDEAL (as an acronym) is used by B. Coll and collaborators long ago. This acronym seems to be adequate if the conditions involved, as well as being Intrinsic and Explicit, are Deductive (no inference process is necessary) and ALgorithmic (a flow chart with a finite number of steps can be built). 
[16].

The accurate analysis of the underlying geometry of the Weyl and Ricci tensors has allowed us to characterize in an ideal form several families of spacetimes which are interesting from the physical and/or geometrical point of view. We can quote the characterizations of the Schwarzschild [17], Reissner-Nordström [18] and Kerr [19] black holes, among others (see references in [20]).

As Takeno claimed, the ideal characterization of spacetimes provides an algorithmic way to test if a metric tensor, given in an arbitrary coordinate system, is a specific solution of Einstein equations. This can be useful to check wether a new solution is an already known one. Also, it can be of interest in obtaining a fully algorithmic characterization of the initial data which correspond to such a solution. For instance, our ideal labeling of the Schwarzschild geometry [17] has allowed us to achieve the algorithmic characterization of the Schwarzschild initial data [21].

A spherically symmetric spacetime is a $2+2$ warped product and this quality determines the algebraic properties of the curvature tensor of a metric with spherical symmetry. The approach that we present here is based on this fact. We start from the ideal characterization of the $2+2$ warped spacetimes recently obtained [20], and we look for the complementary conditions on the curvature tensor which lead to spherical symmetry. Section 2 is devoted to the study of the s-warped metrics, the class of the $2+2$ warped spacetimes containing the spherically symmetric ones: we present their ideal labeling and summarize some properties of their curvature tensor.

In Section 3 we analyze the complementary constraints that the spherical symmetry imposes and, when the Weyl tensor does not vanish, we write these new conditions in terms of explicit concomitant of the Weyl and Ricci tensors. Thus, we obtain our main result: the ideal characterization of the non conformally flat spherically symmetric spacetimes. Further, we give the algorithm to determine the canonical elements of a spherically symmetric spacetime from the Riemann tensor. An alternative characterization which applies for a wide range of Ricci algebraic types is also offered.

In Section 4 we show that our result can be useful to label specific spherically symmetric solutions in an ideal form. As a consequence of our main theorem we recover the already known characterizations of the Schwarzschild and Reissner-Nordström spacetimes [17] [18], and we obtain the ideal labeling of the Lemaitre-Tolman-Bondi solution.

In this paper we work on an oriented spacetime with a metric tensor $g$ of signature $\{-,+,+,+\}$. The Riemann, Weyl and Ricci tensors and the scalar curvature are defined as given in [22] and denoted, respectively, by $\operatorname{Riem}(g), W=\mathrm{W}(g), R=\operatorname{Ric}(g)$ and r. For the metric product of two vectors we write $(x, y)=g(x, y)$, and we put $x^{2}=g(x, x)$. Other basic notation used in this work is summarized in Appendix A. 


\section{2. s-warped spacetimes}

A classification scheme for the warped product spacetimes was proposed by Carot and da Costa [23] and the isometry group that each class can admit was also studied. The energy tensor algebraic types compatible with these classes were later analyzed [24].

In a recent paper [20] we have given an invariant classification of the spacetimes conformal to a $2+2$ product and, for the non conformally flat case, we have characterized each class in an ideal form. The $2+2$ warped metrics correspond to two of these invariant classes, the $t$-warped and the $s$-warped spacetimes.

In the s-warped spacetimes the warped plane is space-like, and the metric tensor takes the expression [20]:

$$
g=v+e^{2 \lambda} \hat{h}, \quad \hat{h}(\mathrm{~d} \lambda)=0,
$$

where $\lambda$ is the warping factor and where $v$ and $\hat{h}$ denote two 2-dimensional metrics, Lorentzian and Riemannian, respectively.

A s-warped spacetime has two principal planes, the time-like $\mathrm{V}$ with projector $v$ and the space-like $\mathrm{H}$ with projector $h=e^{2 \lambda} \hat{h}$. The pair $(v, h)$ defines a $2+2$ almostproduct structure with structure tensor $\Pi=v-h$. A $2+2$ spacetime structure is also determined by the canonical time-like unitary 2-form $U$, volume element of the plane $\mathrm{V}, U^{2}=v$.

\subsection{Invariant characterization of the s-warped spacetimes}

The properties of the principal structure $(v, h)$ offer an invariant characterization of the s-warped metrics [20]. More precisely, the structure is umbilical and integrable, the plane $\mathrm{V}$ is minimal, and the trace of the second fundamental form of the plane $\mathrm{H}$ is a closed 1-form. These differential constraints can be explicitly written in terms of either the canonical 2-form $U$ or the projector $h$ and, consequently, one obtains an invariant labeling of the s-warped metrics [20]:

Proposition 1 A $2+2$ s-warped spacetime is characterized by one of the following equivalent conditions:

(i) There exists a simple, time-like and unitary 2 -form $U\left(U \cdot * U=0, \operatorname{tr} U^{2}=2\right)$ such that

$$
2 \nabla * U=-* U \bowtie b, \quad \mathrm{~d} b=0, \quad b \equiv U(\nabla \cdot U) \neq 0 .
$$

(ii) There exists a space-like 2-projector $h\left(h^{2}=h, \operatorname{tr} h=2, h(x, x) \geq 0\right.$, where $x$ in an arbitrary time-like vector) such that

$$
2 \nabla h=h \stackrel{23}{\widetilde{\otimes}} b, \quad \mathrm{~d} b=0, \quad b \equiv \nabla \cdot h \neq 0 .
$$

Moreover, $U$ is the canonical 2-form and $h$ is the projector on the space-like plane. The warping factor $\lambda$ satisfies $2 \mathrm{~d} \lambda=-b$. 


\subsection{Ricci and Weyl tensors}

If we define $\hat{v}=e^{-2 \lambda} v$, we obtain that a s-warped metric can be written as conformal to a $2+2$ product metric, $g=e^{2 \lambda}(\hat{v}+\hat{h})$, with $\hat{h}(\mathrm{~d} \lambda)=0$. Then, taking into account how the Weyl and Ricci tensors change by a conformal transformation, we have [20]:

Proposition 2 Let $g=e^{2 \lambda}(\hat{v}+\hat{h})$ be a s-warped metric and let $X, Y$ be the Gauss curvatures of $\hat{v}$ and $\hat{h}$. Then,

(i) The Weyl tensor is type $D$ (or $O)$ with double eigenvalue $\rho \equiv-\frac{1}{6} e^{-2 \lambda}(X+Y)$. In the type $D$ case, the canonical 2-form $U, U^{2}=v$, is the principal 2-form of the Weyl tensor which takes the expression:

$$
W=\rho(3 S+G), \quad S \equiv U \otimes U-* U \otimes * U, \quad G \equiv \frac{1}{2} g \oslash g .
$$

(ii) The space-like principal plane is an eigen-plane of the Ricci tensor $R$, that is, $R \cdot h=\mu h$ where $\mu$ is the associated eigenvalue. The Ricci tensor takes the expression:

$$
R=X \hat{v}+Y \hat{h}-2 \nabla \mathrm{d} \lambda-2 \mathrm{~d} \lambda \otimes \mathrm{d} \lambda-[\Delta \lambda-2 g(\mathrm{~d} \lambda, \mathrm{d} \lambda)] g .
$$

Note that the gradient of the warping factor is given by the vector $b$ defined in proposition 1, $2 \mathrm{~d} \lambda=-b$. Then, if we take the trace in expression (5) and we consider the expression of the Weyl eigen-value given in point (i) of proposition 2, we get:

$$
4 \rho+\frac{\mathrm{r}}{3}=\nabla \cdot b+\frac{1}{2} b^{2}
$$

where $\mathrm{r}$ is the scalar curvature, $\rho$ the double Weyl eigenvalue and $b \equiv \nabla \cdot h, h$ being the projector on the space-like plane.

On the other hand, we can obtain the Gauss curvature of the metric $h, \kappa=e^{-2 \lambda} Y$, as follows. Note that $h(b)=0$ and, consequently, $h^{\alpha \beta} \nabla_{\alpha} b_{\beta}=-b^{2}$. Then, if we take the trace with the projector $h$ in expression (5), we obtain:

$$
\kappa=e^{-2 \lambda} Y=\mu-\frac{1}{2} \nabla \cdot b,
$$

where $\mu$ is the Ricci eigenvalue associated with the eigen-plane $\mathrm{H}, 2 \mu=h^{\alpha \beta} R_{\alpha \beta}$. Moreover, if we remove $\nabla \cdot b$ from equations (6) and (7), we can obtain an alternative expression for the Gauss curvature $\kappa$ :

$$
\kappa=-2 \rho-\frac{1}{6} \mathrm{r}+\mu+\frac{1}{4} b^{2} .
$$

\subsection{Ideal characterization of the non conformally flat s-warped metrics}

As a consequence of proposition 2, in the non conformally flat case the Weyl tensor is type $\mathrm{D}$ and the canonical 2-form $U$ can be obtained from the Weyl tensor. Then, the invariant characterization given in proposition 1 leads to the following characterization of the s-warped metrics [20]: 
Proposition 3 For a non conformally flat metric $g$ let $\rho, S$ and $\Phi$ be the Weyl concomitants

$$
\rho \equiv-\left(\frac{1}{12} \operatorname{tr} W^{3}\right)^{\frac{1}{3}} \neq 0, \quad S \equiv \frac{1}{3 \rho}(W-\rho G), \quad \Phi \equiv \operatorname{tr}[S(\nabla \cdot S)] .
$$

Then, $g$ is a $2+2$ s-warped metric if, and only if, it satisfies:

$$
\begin{aligned}
& S^{2}+S=0, \\
& 2 \nabla \cdot S+3 S(\nabla \cdot S)-g \oplus \Phi=0, \quad \mathrm{~d} \Phi=0, \\
& * S(\Phi ; \Phi)=0, \quad 2 S(\Phi, x ; \Phi, x)-\Phi^{2}>0,
\end{aligned}
$$

where $x$ is an arbitrary unitary time-like vector. Moreover the warping factor $\lambda$ satisfies $\Phi=-2 \mathrm{~d} \lambda$.

The notation used in this proposition is explained in detail in Appendix A. Each of the conditions has a specific geometric interpretation. The algebraic condition (10) means that the Weyl tensor is of type $\mathrm{D}$ with real eigenvalues. The two differential conditions (11) ensure that the metric is conformal to a $2+2$ product metric with a principal structure aligned with the Weyl tensor and, moreover, $\Phi=-2 \mathrm{~d} \lambda$. The differential conditions (12) mean that $\Phi$ lies on the time-like principal plane or, equivalently, that $h(\mathrm{~d} \lambda)=0$.

This proposition will be the starting point in obtaining the characterization of the spherically symmetric spacetimes.

\section{Characterizing spherically symmetric spacetimes}

In a canonical coordinate system $(t, r, \theta, \varphi)$, the metric line element of a spherically symmetric spacetime takes the expression [4] [1]:

$$
\mathrm{d} s^{2}=A \mathrm{~d} t^{2}+2 C \mathrm{~d} t \mathrm{~d} r+B \mathrm{~d} r^{2}+e^{2 \lambda} \mathrm{d} \Omega^{2},
$$

where $\mathrm{d} \Omega^{2}=\mathrm{d} \theta^{2}+\sin ^{2} \mathrm{~d} \varphi^{2}$ is the metric of the 2-sphere and $A, B, C$ and $\lambda$ are smooth functions of $t$ and $r$.

In the coordinate expression (13) we can identify the canonical form of a s-warped metric. More precisely, we have:

Lemma 1 A spacetime is spherically symmetric if, and only if, the metric tensor can be written as:

$$
g=v+e^{2 \lambda} \sigma, \quad \sigma(\mathrm{d} \lambda)=0,
$$

where $v$ is an arbitrary 2-dimensional Lorentzian metric, and $\sigma$ is a 2-dimensional Riemannian metric with a Gauss curvature equal to one. 


\subsection{Invariant characterization of the spherically symmetric spacetimes}

In the previous section we have characterized the s-warped spacetimes. Now we must add new constraints in order to impose that $\hat{h}=\sigma, \sigma$ being a metric with Gauss curvature $Y=1$. As a consequence of (7), this condition holds if, and only, if, the Gauss curvature of $h$ is $\kappa=e^{-2 \lambda}>0$.

On the other hand, as $h(\mathrm{~d} \lambda)=0$, the condition $Y=1$ implies $h(\mathrm{~d} \kappa)=0$. And, conversely, as $Y$ is the curvature of the metric $\hat{h}$ and $\kappa=e^{-2 \lambda} Y$, conditions $h(\mathrm{~d} \kappa)=0$ and $\kappa>0$ imply that $Y$ is a positive constant and, consequently, we can add an additive constant to $\lambda$ in order to obtain a metric $\sigma$ with Gauss curvature $Y=1$.

Therefore, a s-product spacetime is spherically symmetric if, and only if, the Gauss curvature $\kappa$ of the metric $h$ of the space-like principal plane satisfies:

$$
\kappa \equiv \kappa(h)>0, \quad h(\mathrm{~d} \kappa)=0 .
$$

Then, if we take into account proposition 1, we obtain an invariant characterization of the metrics with spherical symmetry:

Proposition 4 A spherically symmetric spacetime is characterized by one of the following equivalent conditions:

(i) There exists a simple, time-like and unitary 2-form $U$ which satisfies (2) and (15), where $\kappa(h)$ is the Gauss curvature of the metric $h=U^{2}$.

(ii) There exists a space-like 2-projector $h$ which satisfies (3) and (15), where $\kappa(h)$ is the Gauss curvature of $h$.

Moreover, $U$ is the canonical 2-form and $h$ is the projector on the 2-spheres. The warping

factor $\lambda$ can be obtained as $\lambda=-\frac{1}{2} \ln \kappa$, and $\sigma=\kappa h$ is the metric of the 2-sphere.

\subsection{Ideal characterization of the non conformally flat spherically symmetric spacetimes}

Proposition 4 characterizes the spherically symmetric spacetimes whatever their Riemann algebraic type. In order to obtain an intrinsic labeling we must give either the canonical 2-form $U$ or the projector $h$ in terms the of the Weyl or Ricci tensors. The use of the Ricci tensor requires the analysis of several algebraic types and will be considered elsewhere [26]. Here we use the Weyl tensor.

When the spacetime is not conformally flat $(W \neq 0)$, the Weyl tensor is of type $\mathrm{D}$ and, as stated in proposition 2, the canonical 2-form $U$ is aligned with the Weyl tensor. The explicit expression $U=U[W]$ of this Weyl concomitant is given in Appendix B. Then, as a consequence of proposition 4, if we impose conditions (2) and (15) to the 2-form $U=U[W]$ we get an intrinsic labeling of the non conformally flat spherically symmetric spacetimes. Nevertheless, a more accurate analysis of these constraints leads to more explicit conditions in the Weyl tensor. Indeed, in [20] we have shown that conditions (21) are equivalent to equations (10), (11) and (12) which characterize the s-warped metrics in an ideal form (see proposition 3). Thus, at this point we have: 
Lemma 2 A non conformally flat spacetime is spherically symmetric if, and only if, the metric tensor satisfies the intrinsic and explicit conditions (10), (11) and (12), and the invariant conditions (15).

Now we analyze the conditions (15) in order to complete an ideal characterization of the spherical symmetry.

As commented after proposition 3, constraint (12) states that $h(\Phi)=0$. Moreover, under this condition, the Weyl concomitant $\Phi$ defined in (9) equals the vector $b$ defined in proposition 1. Then, proposition 4 implies that for spherically symmetric spacetimes the following identities hold:

$$
\Phi=-2 \mathrm{~d} \lambda=b=\mathrm{d} \ln \kappa .
$$

Note that the necessary conditions $h(\Phi)=0$ and $\Phi=\mathrm{d} \ln \kappa$ imply that $\mathrm{d} \Phi=0$ and $h(\mathrm{~d} \kappa)=0$. Consequently, we have:

Lemma 3 In the set of constraints (10), (11), (12) and (15) characterizing the spherically symmetric spacetimes we can substitute conditions $\mathrm{d} \Phi=0$ and $h(\mathrm{~d} \kappa)=0$, by the condition $\Phi=\mathrm{d} \ln \kappa$.

Finally, let us analyze expression (8) of the curvature $\kappa$. The scalars $\rho$ and $\mathrm{r}$, and the vector $b=\Phi$ are explicit concomitants of the Weyl and Ricci tensors. How can the Ricci eigenvalue $\mu$ be explicitly obtained? It satisfies $2 \mu=h^{\alpha \beta} R_{\alpha \beta}$, where the projector $h$ could be obtained from the Weyl concomitant $U=U[W]$ given in lemma 7 as $h=g-U^{2}$. Nevertheless, we try to avoid the use of this expression of $U$ in our characterization theorem because it involves an arbitrary 2-form $Z$ which could be annoying in subsequent applications. Now, we get an alternative expression for $\mu$ whatever the Ricci algebraic type by using a mixed concomitant of the Ricci and Weyl tensors as follows.

The generic expression of a Ricci tensor which has $\mathrm{H}$ as an eigen-plane with associated eigenvalue $\mu$ is:

$$
R=A+\mu h,
$$

where $A$ is a symmetric tensor on $\mathrm{V}, A \cdot h=0$. Then, from the expression (41) of $S$, we obtain:

$$
B \equiv R-S[R]-\frac{1}{2} \mathrm{r} g=\beta \Pi, \quad \beta \equiv \frac{1}{2} \mathrm{r}-2 \mu .
$$

Thus, we have $\mu=\frac{1}{4} \mathrm{r}-\frac{1}{2} \beta$, where $\beta=0$ when $B=0$, and when $B \neq 0, \beta$ can be computed as

$$
\beta=\frac{\epsilon}{2} \sqrt{\operatorname{tr} B^{2}}, \quad \epsilon=-\frac{B(x, x)}{\mid(B(x, x) \mid},
$$

$x$ being an arbitrary unitary time-like vector (note that the structure tensor $\Pi=v-h$ satisfies $\Pi(x, x)<0$ for every time-like vector $x)$. This expression of $\beta$ allows us to obtain an explicit expression of the Ricci eigenvalue $\mu$ and, substituting in (8), of the curvature $\kappa$.

All these considerations and lemma 3 lead to the following ideal characterization. 
Theorem 1 Let $W=\mathrm{W}(g)$ and $R=\operatorname{Ric}(g)$ be the Weyl and Ricci tensors of a non conformally flat metric $g$, and let $\rho, S, \Phi, \mathrm{r}, B$ and $\kappa$ be the Riemann concomitants

$$
\begin{aligned}
& \rho \equiv-\left(\frac{1}{12} \operatorname{tr} W^{3}\right)^{\frac{1}{3}} \neq 0, \quad S \equiv \frac{1}{3 \rho}(W-\rho G), \quad \Phi \equiv \operatorname{tr}[S(\nabla \cdot S)], \\
& \mathrm{r} \equiv \operatorname{tr} R, \quad B \equiv R-S[R]-\frac{1}{2} \mathrm{r} g, \quad \kappa \equiv-2 \rho+\frac{1}{12} r+\frac{1}{4} \Phi^{2}+\frac{\epsilon}{4} \sqrt{\operatorname{tr} B^{2}} .
\end{aligned}
$$

where we put either $\epsilon=0$ if $B=0$ or $\epsilon=-\frac{B(x, x)}{|B(x, x)|}$ if $B \neq 0, x$ being an arbitrary unitary time-like vector.

Then, $g$ is spherically symmetric if, and only if, it satisfies:

$$
\begin{aligned}
& S^{2}+S=0, \\
& 2 \nabla \cdot S+3 S(\nabla \cdot S)-g \oplus \Phi=0, \\
& * S(\Phi ; \Phi)=0, \quad 2 S(\Phi, x ; \Phi, x)-\Phi^{2}>0, \\
& \kappa>0, \quad \Phi=\mathrm{d} \ln \kappa,
\end{aligned}
$$

where $x$ is an arbitrary unitary time-like vector.

A metric tensor $g$ given in any coordinate system which satisfies the conditions of theorem 1 is a spherically symmetric solution and, as a consequence of lemma 11 it admits the canonical form (14). Can the canonical elements $v, \sigma$ and $\lambda$ be obtained from the Riemann tensor?

As stated in the last sentence of proposition 4, the warping factor can be obtained from the Gauss curvature $\kappa$ as $\lambda=-\frac{1}{2} \ln \kappa$, and $\sigma=\kappa h$. Moreover $h$ can be obtained as $h=g-v$, and $v=U^{2}$ where $U$ is the Weyl principal 2-form. Thus, we can state:

Proposition 5 A non conformally flat metric $g$ with spherical symmetry admits the canonical form:

$$
g=v+\frac{1}{\kappa} \sigma, \quad v=U^{2},
$$

where $\kappa$ and $U$ are, respectively, the Riemann concomitants defined in (21) and (B.1).

\subsection{On the other characterizations based more closely on the Ricci tensor}

Theorem [1 and proposition 5 apply when the Weyl tensor does not vanish whatever the Ricci algebraic type. Under any algebraic constraint of the Ricci tensor, alternative statements can be deduced. Elsewhere [26] we analyze in detail other characterization theorems based more closely on the Ricci tensor. Here, we illustrate these approaches by imposing an algebraic condition which is valid for a wide range of Ricci types and which avoids the use of the canonical 2-form $U$ in obtaining the canonical elements.

Let us suppose that the 2 -tensor $B$ defined in (18) does not vanish. Then $\beta \neq 0$ and the structure tensor $\Pi$ can be obtained as $\Pi=\frac{1}{\beta} B$. Thus, taking into account that condition (24) is equivalent to $h(\Phi)=0$, we can state: 
Proposition 6 Let $g$ be a non conformally flat metric such that the Riemann concomitant $B$ defined in (18) does not vanish. Then, the metric is spherically symmetric if, and only if, it satisfies (22), (23), (25) and $B(\Phi)=\beta \Phi$, where $\beta$ is given in (19).

Then, the metric $g$ admits the canonical form (26), where $\kappa$ is defined in (21) and $v$ is given by $v=\frac{1}{2}\left(g+\frac{1}{\beta} B\right)$.

\section{Labeling specific spherically symmetric solutions}

The ideal characterization given in theorem 1 allows us to distinguish wether a non conformally flat metric given in any coordinate system is spherically symmetric. Moreover, this result could easily help us to obtain the ideal labeling of specific spherically symmetric solutions. In this section we illustrate this fact by recovering the already known characterizations of the Schwarzschild [17] and Reissner-Nordström [18] geometries, and by obtaining the ideal characterization of the Lemaître-Tolman-Bondi solution.

In the two last cases, with a non vanishing Ricci tensor, an alternative characterization based more closely on Ricci concomitants could be acquired. Nevertheless, here we apply our above theorem 1 and proposition 6 based essentially on the Weyl tensor. This way, the characterization theorem for the Reissner-Nordström admits the Schwarzschild solution as a limiting case.

\subsection{Ideal characterization of the Schwarzschild solution}

The Jebsen-Birkhoff theorem [27] 28] states that the Schwarzschild spacetime is the only spherically symmetric vacuum solution. Consequently, we have:

Lemma 4 A metric tensor $g$ is the Schwarzschild solution if, and only if, it satisfies $R \equiv \operatorname{Ric}(g)=0$ and the conditions of theorem 1 .

Let us see how the conditions in the above lemma can be simplified. If we replace $S$ in terms of the Weyl tensor, condition (23) is equivalent to

$$
\rho \nabla \cdot W+W(\nabla \cdot W)-\frac{1}{3} g \bowtie \omega=0, \quad \omega=\operatorname{tr} W(\nabla \cdot W) .
$$

On the other hand, $\omega$ and $\Phi$ are related as $\Phi=\frac{1}{9 \rho^{2}} \omega+\frac{2}{3} \mathrm{~d} \ln \rho$.

Then, under the vacuum condition, $R=0$, the Cotton tensor vanishes and then $\nabla \cdot W=0$. Consequently, (23) identically holds and $\Phi=\frac{2}{3} \mathrm{~d} \ln \rho$. Then, the second condition in (25) also holds and, moreover, the curvature $\kappa$ takes the expression $\kappa=\frac{1}{9}(\mathrm{~d} \ln \rho)^{2}-2 \rho$. Finally, we can substitute $\Phi$ by $\mathrm{d} \rho$ in equation (24). Thus, we recover the following ideal characterization of the Schwarzschild geometry [17]:

Theorem 2 Let $W=\mathrm{W}(g)$ and $R=\operatorname{Ric}(g)$ be the Weyl and Ricci tensors of a non conformally flat metric $g$, and let $\rho$ and $S$ be the Weyl concomitants

$$
\rho \equiv-\left(\frac{1}{12} \operatorname{tr} W^{3}\right)^{\frac{1}{3}}, \quad S \equiv \frac{1}{3 \rho}(W-\rho G) .
$$


Then, the necessary and sufficient conditions for $g$ to be the Schwarzschild metric are:

$$
\begin{aligned}
& \operatorname{Ric}(g)=0, \quad \rho \neq 0, \quad S^{2}+S=0, \\
& \kappa \equiv \frac{1}{9}(\mathrm{~d} \ln \rho)^{2}-2 \rho>0, \\
& * S(\mathrm{~d} \rho ; \mathrm{d} \rho)=0, \quad 2 S(\mathrm{~d} \rho, x ; \mathrm{d} \rho, x)-(\mathrm{d} \rho)^{2}>0,
\end{aligned}
$$

where $x$ is an arbitrary unitary time-like vector.

\subsection{Ideal characterization of the Reissner-Nordström solution}

As a consequence of the generalized Birkhoff theorem (see [29] and references therein), the only spherically symmetric non null Einstein-Maxwell solution is the ReissnerNordström spacetime. The spherical symmetry implies that the electromagnetic field is aligned with the Weyl tensor and moreover, as the principal structure is integrable, the differential Rainich condition identically holds [30] [31. Then, we only have to add the algebraic Rainich conditions. Consequently, we have:

Lemma 5 A metric tensor $g$ is the Reissner-Nordström solution if, and only if, it satisfies the conditions of theorem 1 and:

$$
\operatorname{tr} R=0, \quad R^{2}=\chi^{2} g, \quad R(x, x) \geq 0,
$$

where $x$ is an arbitrary unitary time-like vector and $\chi \equiv-\frac{1}{2} \sqrt{\operatorname{tr} R^{2}}$.

Again, the conditions in the above lemma can be simplified. As commented above, the spherical symmetry implies the alignment of the Ricci and Weyl tensors, an algebraic condition which is equivalent to:

$$
S[R]+R=0 .
$$

Under this constraint, the second Rainich condition in (31) is a consequence of the type D condition (22). Moreover, in [31] we have shown that the algebraic constraints (22) and (32) imply the differential condition (23) under the complementary algebraic restriction $9 \rho^{2} \neq \chi^{2}$.

On the other hand, for aligned non null Einstein-Maxwell solutions, the Bianchi identities imply (when the Weyl tensor has real eigenvalues) [31]:

$$
\mathrm{d} \chi=2 \chi \Phi, \quad 2 \mathrm{~d} \rho=3 \rho \Phi+\chi \Pi(\Phi) .
$$

The second of these equations implies, under the algebraic condition $9 \rho^{2} \neq \chi^{2}$, that $h(\Phi)=0$ if, and only if, $h(\mathrm{~d} \rho)=0$. Thus, we can substitute $\Phi$ by $\mathrm{d} \rho$ in equation (24). Moreover, in this case $\mu=-\chi$, and from (33) we have $\Phi=\frac{2}{3} \mathrm{~d} \ln (\rho+\chi)$, and we obtain that the curvature $\kappa$ given in (21) becomes $\kappa=\frac{1}{9}(\mathrm{~d} \ln \rho)^{2}-2 \rho-\chi$. Thus, we recover

the following ideal characterization of the Reissner-Nordström geometry [18]:

Theorem 3 Let $W=\mathrm{W}(g)$ and $R=\operatorname{Ric}(g)$ be the Weyl and Ricci tensors of a non conformally flat metric $g$, and let $\rho, S$ and $\chi$ be the Riemann concomitants

$$
\rho \equiv-\left(\frac{1}{12} \operatorname{tr} W^{3}\right)^{\frac{1}{3}}, \quad S \equiv \frac{1}{3 \rho}(W-\rho G), \quad \chi \equiv-\frac{1}{2} \sqrt{\operatorname{tr} R^{2}}
$$


Then, the necessary and sufficient conditions for $g$ to be the Reissner-Nordström metric are:

$$
\begin{aligned}
& \rho \neq 0, \quad S^{2}+S=0, \quad \operatorname{tr} R=0, \quad R(x, x) \geq 0, \\
& S[R]+R=0, \quad 9 \rho^{2} \neq \chi^{2}, \\
& \kappa \equiv \frac{1}{9}(\mathrm{~d} \ln \rho)^{2}-2 \rho-\chi>0, \\
& * S(\mathrm{~d} \rho ; \mathrm{d} \rho)=0, \quad 2 S(\mathrm{~d} \rho, x ; \mathrm{d} \rho, x)-(\mathrm{d} \rho)^{2}>0,
\end{aligned}
$$

where $x$ is an arbitrary unitary time-like vector.

If we make $\chi=0$ in the above theorem we recover the conditions of theorem 2 which characterize the Schwarzschild solution. Thus, theorem 3 includes the vacuum case.

\subsection{Ideal characterization of the Lemaître-Tolman-Bondi solution}

The Lemaître-Tolman-Bondi metrics (see, for example [22]) are the spherically symmetric dust solutions such that the gradient of the warped factor $\lambda$ is not collinear with the velocity of the fluid. These constraints and the energy condition can be explicitly written in terms of the Ricci tensor. Then we obtain:

Lemma 6 A metric tensor $g$ is the Lemaitre-Tolman-Bondi solution if, and only if, it satisfies the conditions of theorem 1 and:

$$
R^{2}=\frac{1}{4} \mathrm{r}^{2} g, \quad \mathrm{r} \equiv \operatorname{tr} R>0, \quad T \oplus \Phi \neq 0
$$

where $T \equiv R-\frac{1}{2} \mathrm{r} g$.

We can simplify the conditions in this lemma considering the alternative characterization given in proposition [6 since now $B=-\frac{\mathrm{r}}{2} \Pi \neq 0$. Then $\beta=-\frac{\mathrm{r}}{2}$, and the curvature $\kappa$ takes the expression $\kappa=-2 \rho+\frac{1}{4} \Phi^{2}-\frac{1}{6} \mathrm{r}$. Thus, we obtain the following ideal characterization of the Lemaître-Tolman-Bondi geometry:

Theorem 4 Let $W=\mathrm{W}(g)$ and $R=\operatorname{Ric}(g)$ be the Weyl and Ricci tensors of a non conformally flat metric $g$, and let $\rho, S, \Phi, \mathrm{r}, B$ and $\kappa$ be the Riemann concomitants

$$
\begin{aligned}
& \rho \equiv-\left(\frac{1}{12} \operatorname{tr} W^{3}\right)^{\frac{1}{3}} \neq 0, \quad S \equiv \frac{1}{3 \rho}(W-\rho G), \quad \Phi \equiv \operatorname{tr}[S(\nabla \cdot S)], \\
& \mathrm{r} \equiv \operatorname{tr} R, \quad B \equiv R-S[R]-\frac{1}{2} \mathrm{r} g, \quad T \equiv R-\frac{1}{2} \mathrm{r} g .
\end{aligned}
$$

Then, the necessary and sufficient conditions for $g$ to be the Lemaître-Tolman-Bondi metric are:

$$
\begin{aligned}
& \rho \neq 0, \quad S^{2}+S=0, \quad \mathrm{r}>0, \quad R^{2}=\frac{1}{4} \mathrm{r}^{2} g, \quad T \oplus \Phi \neq 0, \\
& 2 \nabla \cdot S+3 S(\nabla \cdot S)-g \oplus \Phi=0, \quad B(\Phi)+\frac{\mathrm{r}}{2} \Phi=0, \\
& \kappa \equiv-2 \rho+\frac{1}{4} \Phi^{2}-\frac{1}{6} \mathrm{r}>0, \quad \Phi=\mathrm{d} \ln \kappa .
\end{aligned}
$$


Characterization of spherically symmetric spacetimes

\section{Acknowledgments}

This work has been supported by the Spanish Ministerio de Ciencia e Innovación, MICIN-FEDER project FIS2009-07705.

\section{Appendix A. Notation}

(i) Products and other formulas involving 2-tensors $A$ and $B$ :

(a) Composition as endomorphisms: $A \cdot B$,

$$
(A \cdot B)_{\beta}^{\alpha}=A_{\mu}^{\alpha} B_{\beta}^{\mu}
$$

(b) Square and trace as an endomorphism:

$$
A^{2}=A \cdot A, \quad \operatorname{tr} A=A_{\alpha}^{\alpha} .
$$

(c) Action on a vector $x$, as an endomorphism $A(x)$, and as a quadratic form $A(x, x)$ :

$$
A(x)^{\alpha}=A^{\alpha}{ }_{\beta} x^{\beta}, \quad A(x, x)=A_{\alpha \beta} x^{\alpha} x^{\beta} .
$$

(d) Exterior product as double 1-forms: $A \oplus B$ is the double 2-form,

$$
(A \oplus B)_{\alpha \beta \mu \nu}=A_{\alpha \mu} B_{\beta \nu}+A_{\beta \nu} B_{\alpha \mu}-A_{\alpha \nu} B_{\beta \mu}-A_{\beta \mu} B_{\alpha \nu} .
$$

(e) Exterior product with a vector $x$ as double 1-forms: $A \oplus x$ is the vector-valued 2-form,

$$
(A \oplus x)_{\alpha, \mu \nu}=A_{\alpha \mu} x_{\nu}-A_{\alpha \nu} x_{\mu}
$$

(f) Symmetrized tensorial product with a vector $x$ as double 1-forms: $A \stackrel{23}{\otimes} x$ is the vector-valued symmetric 2-tensor,

$$
(A \stackrel{23}{\otimes} x)_{\alpha, \mu \nu}=A_{\alpha \mu} x_{\nu}+A_{\alpha \nu} x_{\mu} .
$$

(ii) Products and other formulas involving double 2-forms $P$ and $Q$ :

(a) Composition as endomorphisms of the 2-forms space: $P \circ Q$,

$$
(P \circ Q)_{\rho \sigma}^{\alpha \beta}=\frac{1}{2} P_{\mu \nu}^{\alpha \beta} Q_{\rho \sigma}^{\mu \nu}
$$

(b) Square and trace as an endomorphism of the 2-forms space:

$$
P^{2}=P \circ P, \quad \operatorname{tr} P=\frac{1}{2} P_{\alpha \beta}^{\alpha \beta} .
$$

(c) Action on a 2-form $X$, as an endomorphism $P(X)$, and as a quadratic form $P(X, X)$,

$$
P(X)_{\alpha \beta}=\frac{1}{2} P_{\alpha \beta}^{\mu \nu} X_{\mu \nu}, \quad P(X, X)=\frac{1}{4} P^{\alpha \beta \mu \nu} X_{\alpha \beta} X_{\mu \nu} .
$$

(d) The Hodge dual operator is defined as the action of the, metric volume element $\eta$ on a 2 -form $F$ and a double 2-form $W$ :

$$
* F=\eta(F), \quad * W=\eta \circ W .
$$

(e) Action on two vectors $x$ and $y, P(x ; y)$,

$$
P(x ; y)_{\alpha \beta}=P_{\alpha \mu \beta \nu} x^{\mu} y^{\nu} .
$$


(f) Action on a vector-valued 2-form $Y$ as an endomorphism $P(Y)$,

$$
P(Y)_{\lambda, \alpha \beta}=\frac{1}{2} P_{\alpha \beta}^{\mu \nu} Y_{\lambda, \mu \nu}
$$

(g) The trace of a vector-valued 2-form $Y$ is the 1 -form $\operatorname{tr} Y$,

$$
(\operatorname{tr} Y)_{\alpha}=g^{\lambda \mu} Y_{\lambda, \mu \alpha}
$$

(h) Action on a symmetric 2-tensor $B$ as an endomorphism $P[B]$,

$$
P[B]_{\alpha \beta}=P_{\alpha}{ }_{\beta}^{\mu}{ }^{\nu} B_{\mu \nu}
$$

\section{Appendix B. Covariant determination of the canonical 2-form $U$ of a type D Weyl tensor with real eigenvalues}

The explicit expression of the canonical 2-form $U$ of a type D Weyl tensor using real formalism has been given in a recent paper [19]. Here we particularize it for the case of real eigenvalues:

Lemma 7 For a Petrov-Bel type D Weyl tensor with real eigenvalue $\rho \equiv-\left(\frac{1}{12} \operatorname{tr} W^{3}\right)^{\frac{1}{3}}$, the canonical 2-form $U$ can be obtained as:

$$
U=U[W] \equiv \frac{1}{\chi \sqrt{\chi+f}}((\chi+f) F+\tilde{f} * F) ; \quad F \equiv P(Z),
$$

where $Z$ is an arbitrary 2 -form and

$$
P \equiv W-\rho G, \quad \chi \equiv \sqrt{f^{2}+\tilde{f}^{2}}, \quad f \equiv \operatorname{tr} F^{2}, \quad \tilde{f} \equiv \operatorname{tr}(F \cdot * F) .
$$

\section{References}

[1] Plebański J and Krasiński A 2006 An introduction to General Relativity and Cosmology (Cambridge U. P.)

[2] Romero J V, Ibáñez J M, Martí J M and Miralles J A 1996 Astrophys. J. 462839

[3] Takeno H 1952 Prog. Theor. Phys. 6317

[4] Takeno H 1966 The Theory of Spherically Symmetric Space-Times (Revised ed.) Scientific Reports of the Research Institute for Theoretical Physiscs Hiroshima University No. 5

[5] Cartan E 1946 Leçons sur le Géométrie des Espaces de Riemann (Gauthier-Vilar, Paris)

[6] Riemann B 1867 Abhandlungen der Königlichen Gesellschaft der Wissenschaften zu Göttingen 13272

[7] Bianchi L 1902 Lezioni di geometria differenziale vol 2 (Spoerri, Pisa)

[8] Cotton É 1899 Annales de la faculté des sciences de Toulouse 2e série 1385

[9] Weyl H 1918 Mathematische Zeitschrift 2384

[10] Schouten J A 1921 Mathematische Zeitschrift 1158

[11] Churchill R V 1932 Trans. Am. Math. Soc 34784

[12] Plebański J 1964 Acta Phys. Polon. B 11579

[13] Bona C, Coll B and Morales J A 1992 J. Math. Phys. 33670

[14] Petrov A Z 1954 Sci. Not. Kazan Univ. 114 55. This article has been reprinted in 2000 Gen. Rel. Grav. 321665

[15] Bel L 1962 Cah. de Phys. 16 59. This article has been reprinted in 2000 Gen. Rel. Grav. 322047 (2000)

[16] Ferrando J J, Morales J A and Sáez J A 2001 Class. Quantum Grav. 184969

[17] Ferrando J J and Sáez J A 1998 Class. Quantum Grav. 151323 
[18] Ferrando J J and Sáez J A 2004 J. Math. Phys. 45652

[19] Ferrando J J and Sáez J A 2009 Class. Quantum Grav. 26075013

[20] Ferrando J J and Sáez J A 2010 (submitted to Class. Quantum Grav.) (arXiv:1005.1491v1[gr-qc])

[21] García-Parrado Gómez-Lobo A and Valiente Kroon J A 2007 Phys. Rev. D 75024027

[22] Stephani E, Kramer H, McCallum M A H, Hoenselaers C and Hertl E 2003 Exact Solutions of Einstein's Field Equations (Cambridge University Press, Cambridge)

[23] Carot J and da Costa J 1993 Class. Quantum Grav. 10461

[24] Haddow B and Carot J 1996 Class. Quantum Grav. 13289

[25] Ferrando J J and Sáez J A 2007 J. Math. Phys. 48102504

[26] Ferrando J J and Sáez J A 2010 Characterizing spherically symmetric spacetimes with the Ricci tensor (in preparation)

[27] Jebsen J T 1921 Ark.Mat. Ast. Fys. 73 261. English translation in 2005 Gen. Rel. Grav. 372253

[28] Birkhoff G D 1923 Relativity and Modern Physics (Cambridge, MA: Harvard University Press)

[29] Bona C A 1988 J. Math. Phys. 291440

[30] Rainich G Y 1925 Trans. Math. Soc. 27106

[31] Ferrando J J and Sáez J A 2007 Gen. Relativ. Gravit. 392039 